\title{
Analysis of chaotic motion and its shape dependence in a generalized piecewise linear map
}

\author{
S. Rajagopalan 目and M. Sabir 7 \\ Department of Physics, \\ Cochin University of Science and Technology, \\ Cochin 682 022, Kerala, India.
}

\begin{abstract}
We analyse the chaotic motion and its shape dependence in a piecewise linear map using Fujisaka's characteristic function method. The map is a generalization of the one introduced by R. Artuso. Exact expressions for diffusion coefficient are obtained giving previously obtained results as special cases. Fluctuation spectrum relating to probability density function is obtained in a parametric form. We also give limiting forms of the above quantities. Dependence of diffusion coefficient and probability density function on the shape of the map is examined.

PACS numbers: (i) 05.45. -a (ii) 05.40. -a (iii) 05.60. -k (iv) 45.05. +x
\end{abstract}

\footnotetext{
* Permanent Address: Department of Physics, Sreekrishna College, Guruvayur 680 102, Kerala, India.

e-mail: srgopal9999@yahoo.com

$\dagger$ †-mail: msr@cusat.ac.in phone: 0484-540404 Fax:0484-532495
} 
Deterministic diffusion is a well known phenomenon in spatially extended 1-D maps [1-8]. It has been proposed as a possible mechanism to account for the behaviour of Josephson junctions in the presence of microwave radiation [9] and of parametrically driven oscillators [10]. In Hamiltonian dynamics [11] also, transport due to chaos is significant because of its applications in celestial mechanics, confinement problems and so on. Recently, some exactly solvable models have been analysed [6-8]. The only aim in these studies is the evaluation of the exact diffusion coefficient using a cycle expansion technique [12]. It is a well known fact that the chaotic dynamics in spatially extended maps has two complementary aspects - diffusion and intermittency. These are related to the probability distribution which is approximately Gaussian by central limit theorem. Fujisaka's characteristic function method is a useful tool for analyzing both these aspects of stochasticity in such maps. In this brief report, we apply the characteristic function formalism [13-14] to analyse the chaotic motion in a generalized piecewise linear (GPL) map with a variable shape. It is a generalization of the exactly solvable model in ref.[6] allowing analytical study. Exact expression for diffusion coefficient and a parametric representation for the fluctuation spectrum relating to the probability density function (PDF) are obtained. Generalization permits the study of the dependence of these quantities on the shape of the map. We notice that GPL map with flat peaks is more suited to describe systems exhibiting intermittency in time. The generalization brings the map in ref.[6] nearer to sinusoidal maps studied numerically in ref.[1]. A similar shape dependent piecewise linear model has been examined in ref.[2] from the point of view of correlation times.

Chaos-induced diffusion systems have a general form $[4,13]$

$$
X_{t+1}=X_{t}+P_{r}\left(X_{t}\right)=Y_{r}\left(X_{t}\right), \quad P_{r}(X+1)=P_{r}(X)
$$

where $r$ is a control parameter. The sinusoidal map $P_{r}(X)=r \sin (2 \Pi X)$ is an example [1]. After the decomposition $X_{t}=N_{t}+x_{t}$ where $N_{t}$ is the cell number measured in which $X_{t}$ is located and $x_{t}\left(0 \leq x_{t}<1\right)$ the distance measured from the relative origin $X=N_{t}$, 
eq.(1) can be uniquely rewritten as two dynamical laws:

$$
N_{t+1}=N_{t}+\Delta\left(x_{t}\right), \quad x_{t+1}=f\left(x_{t}\right)
$$

Here $\Delta(x)$ is the jumping number defined as the largest integer smaller than $x+P_{r}(x)$ and is free from $N_{t}$ and $f(x)=x+P_{r}(x)-\Delta(x)$, satisfying $0 \leq f(x)<1 . f(x)$ is the reduced map of the extended map (1).

We analyse a piecewise linear map with variable shape of the type in fig.(1). In the general case, the map consists of linear segments with slopes $\pm m_{i}, i=0,1, \cdots, h, m_{i}<m_{i-1}$. For the cells on the bisector, the slope magnitude is $m_{0}$. For the $i^{\text {th }}$ cell above and below this cell on bisector, the slope magnitude changes to $m_{i}$. The reduced map consists of $k=$ $4 h+3$ linear segments. For $k$ increasing from 1 to $4 h+3$, these line segments have slopes $m_{0}, m_{1}, m_{2}, \cdots m_{h},-m_{h},-m_{h-1}, \cdots 0,-m_{1},-m_{2}, \cdots-m_{h}, m_{h}, m_{h-1}, \cdots m_{2}, m_{1}, m_{0}$, and $m_{i} s$ satisfy the relation

$$
\frac{3}{m_{0}}+\sum_{i=1}^{h} \frac{4}{m_{i}}=1
$$

The extended map can be generated from the reduced map by giving suitable jump numbers $\Delta(x)$ (constant for a line segment). These (from left) are $0,1,2, \cdots h, h, h-$ $1, \cdots 2,1,0,-1,-2, \cdots,-h,-h,-(h-1), \cdots-2,-1,0$. Figs.(1) and (2) show the map and the reduced map for $h=1$.

The map (1) can be studied using the characteristic function formalism [13-14]. In this, the dynamics of $A_{t}$ governed by $A_{t+1}=B\left(x_{t}\right) A_{t}(t=0,1,2, \cdots)$ with $A_{0}=1$ is studied. $B\left(x_{t}\right)$ is a steady function of $x_{t}$ which evolves according to the chaotic map $x_{t+1}=f\left(x_{t}\right)\left(0 \leq x_{t}<1\right)[13]$. Equivalently, one can consider the dynamics of the local time average of a time series $\alpha_{t}=\frac{1}{t} \sum_{j=1}^{t} \ln B\left(x_{j}\right)$ [14]. Map (1) can be treated by putting $A_{t}=\exp \left(N_{t}-N_{0}\right) ; B(x)=\exp (\Delta(x))$. We put $N_{0}=0$. Then $\alpha_{t}=\frac{N_{t}}{t}$. The long time dynamics of $N_{t}$ can be studied using Fujisaka's characteristic function

$$
\lambda_{q}=\frac{1}{q} \lim _{t \rightarrow \infty} \frac{1}{t} \ln \left[\left\langle\exp \left(q N_{t}\right)\right\rangle\right]
$$


$\left\langle\exp \left(q N_{t}\right)\right\rangle$ is the average over a steady ensemble and is the q-order moment of $\exp \left(N_{t}\right)$. One can expand $\lambda_{q}$ in the series of cumulants. The expansion converges for $|q|<c, c$ being the convergence radius. In this case, $\lambda_{q}$ can be approximated as

$$
\begin{aligned}
& \lambda_{q}=\lambda_{0}+D q \\
& \lambda_{0}=\alpha_{\infty}=\lim _{t \rightarrow \infty} \frac{N_{t}}{t}
\end{aligned}
$$

where $\lambda_{0}$ is the drift velocity. $D$ is the diffusion coefficient given by

$$
\sigma_{t}=\left\langle\left(N_{t}-\lambda_{0} t\right)^{2}\right\rangle \approx 2 D t
$$

for large values of $t$. $\sigma_{t}$ is the variance of $N_{t}$. Asymptotic PDF of $\alpha_{t}$ has a Gaussian component (central limit theorem) and a non-Gaussian component. For $|q|<<c$, the moment $<\exp \left(q N_{t}\right)>$ is determined by the Gaussian component (diffusion branch of $q$ ) and for $|q|>>c$, it is determined by the non-Gaussian component (intermittency branch of $q$ ). The $\operatorname{PDF} \rho_{t}(\alpha)$ that $\alpha_{t}$ takes values between $\alpha$ and $\alpha+d \alpha$ can be obtained as

$$
\rho_{t}(\alpha) \sim \exp [-\sigma(\alpha) t]
$$

$\sigma(\alpha)$ being the fluctuation spectrum, $\rho_{t}(\alpha) \rightarrow \delta\left(\alpha-\alpha_{\infty}\right)$ as $t \rightarrow \infty . \rho_{t}(\alpha)$ can be obtained from $\lambda_{q}$ in parametric form using the Legendre transform

$$
\begin{aligned}
\alpha & =\frac{d}{d q}\left(q \lambda_{q}\right) \\
\sigma(\alpha) & =q^{2} \frac{d}{d q} \lambda_{q}
\end{aligned}
$$

We first consider the case with $h=1$. The reduced map consists of 7 line segments with slopes (from left) $m_{0}, m_{1},-m_{1},-m_{0},-m_{1}, m_{1}, m_{0}$. These satisfy (3). The FrobeniusPerron operator $\mathbf{H}$ is defined by

$$
\mathbf{H} G(x)=\sum_{k=1}^{7} \frac{G\left(y_{k}\right)}{\left|f^{\prime}\left(y_{k}\right)\right|}=\sum_{k=1}^{7} \frac{G\left(y_{k}\right)}{\left|m_{k}\right|}
$$

where $y_{k}$ is the $k^{t h}$ solution of $f\left(y_{k}\right)=x$ and $f^{\prime}(x)=\frac{d}{d x} f(x) \cdot m_{k}$ is the slope of the $k^{t h}$ line segment of the reduced map. From eq.(9) we note that the invariant density $p^{*}(x)$ is 
uniform $\left(p^{*}(x)=1\right)$ in the interval $0 \leq x \leq 1\left[\mathbf{H} p^{*}(x)=p^{*}(x)\right]$. The Lyapunov exponent $\lambda$ can be obtained as

$$
\lambda=\frac{3}{m_{0}} \ln \left(m_{0}\right)+\frac{4}{m_{1}} \ln \left(m_{1}\right)
$$

Since $m_{i}>1$, we note that $\lambda>0$ and therefore the reduced map is always chaotic. Characteristic function $\lambda_{q}$ can be evaluated [13] using the linear operator defined by Mori et al $[15]$

$$
\begin{gathered}
\widehat{\mathbf{H}} F(x)=\frac{1}{p^{*}(x)} \mathbf{H}\left[p^{*}(x) F(x)\right] \\
\lambda_{q}=\frac{1}{q} \lim _{t \rightarrow \infty} \frac{1}{t} \ln \langle e^{q \Delta x} \underbrace{\widehat{\mathbf{H}} e^{q \Delta x} \widehat{\mathbf{H}} e^{q \Delta x} \cdots \widehat{\mathbf{H}} e^{q \Delta x}}_{t-1}\rangle
\end{gathered}
$$

For our model $\mathbf{H}=\widehat{\mathbf{H}} . \Delta(x)$ which are constant over a line segment are (from left) $0,+1,+1,0,-1,-1,0$. Hence we get from Eqs.(9), (11) and (12)

$$
\begin{aligned}
& \widehat{\mathbf{H}} e^{q \Delta x}=\frac{3}{m_{0}}+\frac{2}{m_{1}} e^{q}+\frac{2}{m_{1}} e^{-q} \\
&=\frac{3}{m_{0}}+\frac{4}{m_{1}} \cosh (q) \\
& \lambda_{q}=\frac{1}{q} \ln \left[\frac{3}{m_{0}}+\frac{4}{m_{1}} \cosh (q)\right]
\end{aligned}
$$

The result can be generalized for integer values of $h$. Again, slopes satisfy relation (3). Frobenius-Perron operator again leads to uniform invariant density $p^{*}(x)=1$. Lyapunov exponent $\lambda$ and characteristic function $\lambda_{q}$ are given by

$$
\begin{aligned}
\lambda & =\frac{3}{m_{0}} \ln \left(m_{0}\right)+\sum_{i=1}^{h} \frac{4}{m_{i}} \ln \left(m_{i}\right) \\
\lambda_{q} & =\frac{1}{q} \ln \left[\frac{3}{m_{0}}+\sum_{i=1}^{h} \frac{4}{m_{i}} \cosh (i q)\right]
\end{aligned}
$$

Map is fully chaotic since $\lambda>0$. The drift velocity $\lambda_{0}=0$, always. The diffusion coefficient $D$ is 


$$
D=\lim _{q \rightarrow 0} \frac{d}{d q} \lambda_{q}=\sum_{i=1}^{h} \frac{2 i^{2}}{m_{i}}
$$

The fluctuation spectrum $\sigma(\alpha)$ can be got in the parametric form using Eq.(8)

$$
\begin{aligned}
\alpha & =\frac{\sum_{i=1}^{h} \frac{4 i}{m_{i}} \sinh (i q)}{\frac{3}{m_{0}}+\sum_{i=1}^{h} \frac{4}{m_{i}} \cosh (i q)} \\
\sigma(\alpha) & =\frac{\sum_{i=1}^{h} \frac{4 i}{m_{i}} \sinh (i q)}{\frac{3}{m_{0}}+\sum_{i=1}^{h} \frac{4}{m_{i}} \cosh (i q)}-\ln \left[\frac{3}{m_{0}}+\sum_{i=1}^{h} \frac{4}{m_{i}} \cosh (i q)\right]
\end{aligned}
$$

$q=0$ gives $\alpha=0 ; \sigma(\alpha)=0$. If $+q$ gives $+\alpha,-q$ will give $-\alpha$ without changing $\sigma(\alpha)$. It can also be noted that maximum value of $\alpha$ is obtained by putting $q \rightarrow \infty$. We have

$$
\alpha_{\max }=h \quad \sigma\left(\alpha_{\max }\right)=\ln \left(\frac{m_{h}}{2}\right)
$$

In the special case when all $m_{i}$ 's are equal $\left(=m_{0}\right)$, eq.(17) can be summed to obtain a closed form expression for $D$. In this case, eq.(3) gives $m_{0}=3+4 h$.

$$
D=\frac{h(h+1)(2 h+1)}{3(4 h+3)}
$$

With $h+1=\beta$,

$$
D=\frac{(\beta-1) \beta(2 \beta-1)}{3(4 \beta-1)}
$$

which reduces to $D=2 / 7$ for $\beta=2$ giving results obtained previously in ref.(6).

Closed form expression can be got also for the special case $m_{i} / m_{i-1}=r=$ a constant, $0<r<1$. Then $m_{i}=m_{0} r^{i}$. From eq.(3)

$$
m_{0}=3+\frac{4\left(1-r^{h}\right)}{r^{h}(1-r)}
$$

For every $r$ between 0 and 1, the model becomes an exactly solvable case. From eq.(17)

$$
D=\frac{2\left[h^{2}+\left(1-2 h-2 h^{2}\right) r+(h+1)^{2} r^{2}-r^{h+1}-r^{h+2}\right]}{\left[3 r^{h}(1-r)+4\left(1-r^{h}\right)\right](1-r)^{2}}
$$

Limiting forms of the above quantities can be obtained for a constant $h$ as the peak shape becomes maximum flat. These can be arrived at by taking limit $r \rightarrow 0$. From eq.(23), $D$ behaves like 


$$
D=\frac{h^{2}}{2}
$$

The above limit can also be obtained by putting $m_{i} \rightarrow \infty(i=0, \cdots h-1)$ and $m_{h} \rightarrow 4$. Applying this we get the following limits

$$
\begin{gathered}
\lim _{r \rightarrow 0} \lambda_{q}=\frac{1}{q} \ln [\cosh (q h)] \\
\lim _{r \rightarrow 0} \alpha=h \tanh (q h) \\
\lim _{r \rightarrow 0} \sigma(\alpha)=\ln \left[\frac{h^{2}-\alpha^{2}}{h^{2}}\right]^{1 / 2}\left[\frac{h+\alpha}{h-\alpha}\right]^{\alpha / 2 h}
\end{gathered}
$$

In fig.(3) we plot diffusion coefficient versus $r$ for $h=2$. It can be observed that $D$ increases with increasing flatness of the peak shape. $D$ varies from 0.9 to 2 when $r$ is varied from 1 to 0 . Increasing $h$, keeping $m_{i}=m_{0}(i=1,2 \cdots h)$ appears to have more influence on increasing $D$. This is because $D=2 / 7=0.29$ for $h=1$, whereas it goes to 0.909 for $h=3$ (eq. (20).

The probability distribution function for $N_{t}$, the distance from the origin, can be obtained using the fluctuation spectrum $\sigma(\alpha)$. From eq.(7), we have

$$
\rho_{t}(N) \sim \frac{1}{t} \exp \left[-\sigma\left(\frac{N}{t}\right) t\right]
$$

$\rho_{t}(N)$ being the PDF that $N_{t}$ takes values between $N$ and $N+d N$. This PDF is approximately Gaussian by central limit theorem. In the exactly normal case $\lambda_{q}$ is given by eq.(5) and $\sigma(\alpha)$ takes the form

$$
\sigma(\alpha)=\frac{\left(\alpha-\lambda_{0}\right)^{2}}{4 D}
$$

with

$$
\alpha=\lambda_{0}+2 D q
$$

For the present model $\lambda_{0}=0$. In fig.(4) we plot $\sigma(\alpha)$ for different maps and compare with the Gaussian form given in eq.(29). For a constant $h$, non-Gaussian character increases 
with increasing flatness of the map. But, as in the case of diffusion coefficient, increasing $h$ has more influence in producing non-Gaussian character of the PDF.

To conclude, analysis of the PDF with fluctuation spectrum brings out that intermittency and non-Gaussian character of the PDF increases with increasing peak height and flatness of the map, height exercising more effect. This is important when one selects models for describing experiments relating to diffusion. For example, systems exhibiting chaotic motion similar to Brownian motion should have a Gaussian distribution. Maps with linear segments having constant slope and minimum peak height are useful in cases like this. Maps with greater height with peaks becoming more flat will be best suited in describing diffusion systems showing intermittency in time. With flatness becoming maximum, diffusion coefficient behaves like $\frac{h^{2}}{2}, h$ being the peak height. Corresponding limiting forms for characteristic function and fluctuation spectrum are also obtained. The limiting form of fluctuation spectrum is quite different from the Gaussian form following from central limit theorem.

We are attempting a generalization of maps with fractional heights given in ref.(7) along similar lines. This work will be reported elsewhere.

We are grateful to University Grants Commission, India, for providing financial assistance through DSA and COSIST schemes. 


\section{REFERENCES}

[1] T. Geisel and J. Nierwetberg, Phys. Rev. Lett. 48, 7 (1982); M. Schell, S. Fraser and R. Kapral, Phys. Rev. A 26, 504 (1982); H. Fujisaka and S. Grossmann, Z. Phys. B 48, $261(1982)$

[2] S. Grossmann and S. Thomae, Phys. Lett. A 97, 263 (1983)

[3] M. Inoue and H. Koga, Prog. Theor. Phys. 68, 2184 (1982)

[4] H. G. Schuster, Deterministic chaos (Physik-Verlag, Weinheim) Chapter 2 (1984)

[5] T. Geisel Deterministic diffusion - A quality of chaos in Non-equilibrium Co-operative Phenomena in Physics and related fields, Ed. M. G. Velarde (Plenum Press, New York) (1984)

[6] R. Artuso, Phys. Lett. A 160, 528 (1991)

[7] H. C. Tseng et al, Phys. Lett. A 195, 74 (1994)

[8] Chia-Chu Chen, Phys. Rev. E 51, 2815 (1995)

[9] B. A. Huberman, J. P. Crutchfield and N. H. Packard, Appl. Phys. Lett. 37, 750 (1980); M. Cirillo and N. F. Pedersen, Phys. Lett. A 90, 150 (1982); E. Ben-Jacob et al, Phys. Rev. Lett. 49, 1599 (1982)

[10] R. W. Leven and B. P. Koch, Phys. Lett. A 86, 71 (1981)

[11] B. V. Chirikov, Phys. Rep. 52, 263 (1979)

[12] R. Artuso, E. Aurell and P. Cvitanovic, Nonlinearity 3, 325 (1990)

[13] H. Fujisaka, Prog. Theor. Phys. 71, 513 (1984)

[14] H. Fujisaka and M. Inoue, Prog. Theor. Phys. 77, 1334 (1987); H. Fujisaka and M. Inoue, Prog. Theor. Phys. 79, 758 (1988); H. Fujisaka, R. Hagihara and M. Inoue, Phys. Rev. A 38, 3680 (1988); H. Fujisaka, A. Yamaguchi and M. Inoue, Prog. Theor. 
Phys. 81, 1146 (1989); H. Shibata, S. Ando and H. Fujisaka, Phys. Rev. A 45, 7049 (1992)

[15] H. Mori, B. C. So and T. Ose, Prog. Theor. Phys. 66, 1266 (1981) 


\section{FIGURE CAPTIONS}

- Fig. 1. Generalised Piecewise Linear (GPL) map with a variable shape with $h=1$. On both axes, units are arbitrary.

- Fig. 2. Reduced map of GPL map in fig.(1). On both axes, units are arbitrary.

- Fig. 3. Variation of Diffusion coefficient $D$ with $r$ for $h=2$. On both axes, units are arbitrary.

- Fig. 4. Fluctuation Spectrum $\sigma(\alpha)$ versus $\alpha$ for different cases. Solid lines represent actual $\sigma(\alpha)$ while dotted lines give corresponding Gaussian forms. (a) $h=1, m_{0}=$

$m_{1}=7(\mathrm{~b}) h=1, m_{0}=100, m_{1}=4.1237$ (c) $h=2, m_{0}=m_{1}=m_{2}=11$. On both axes, units are arbitrary. 


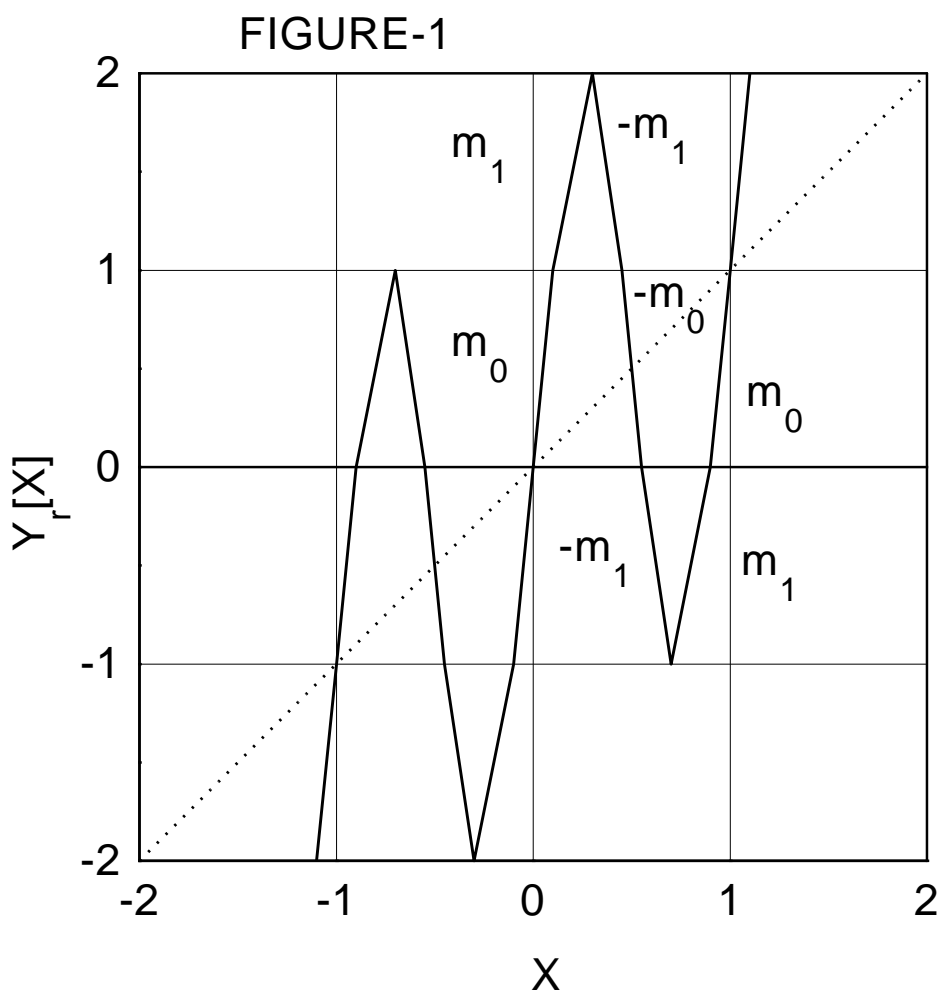




\section{FIGURE-2}

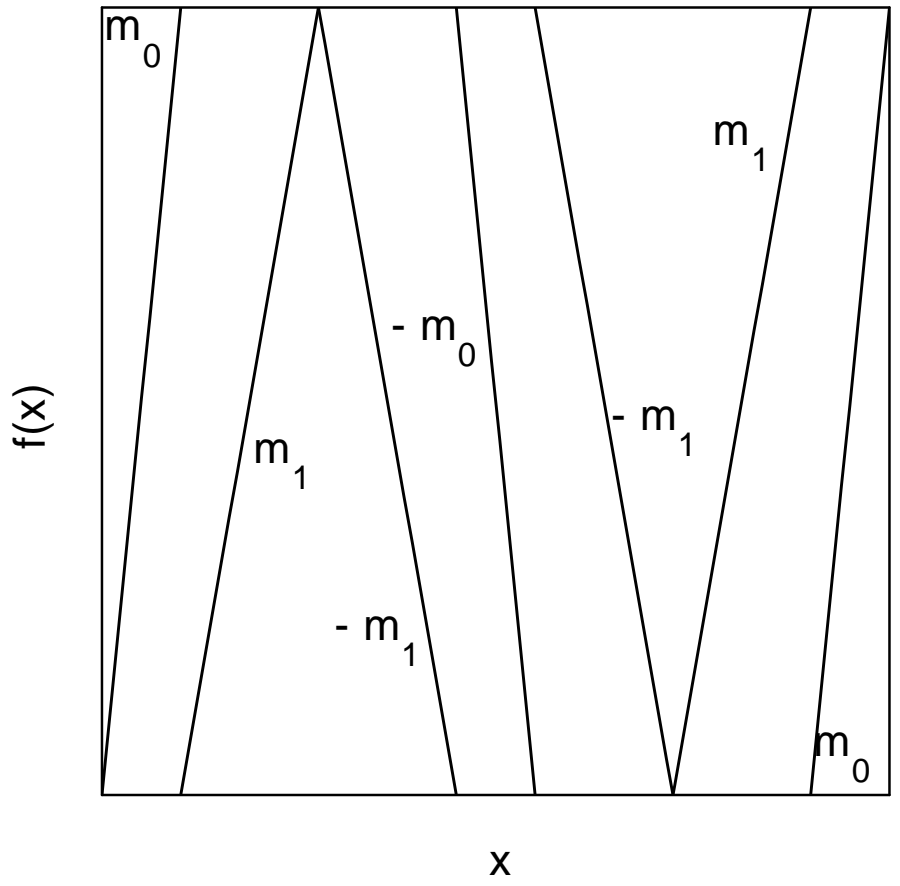


FIGURE-3

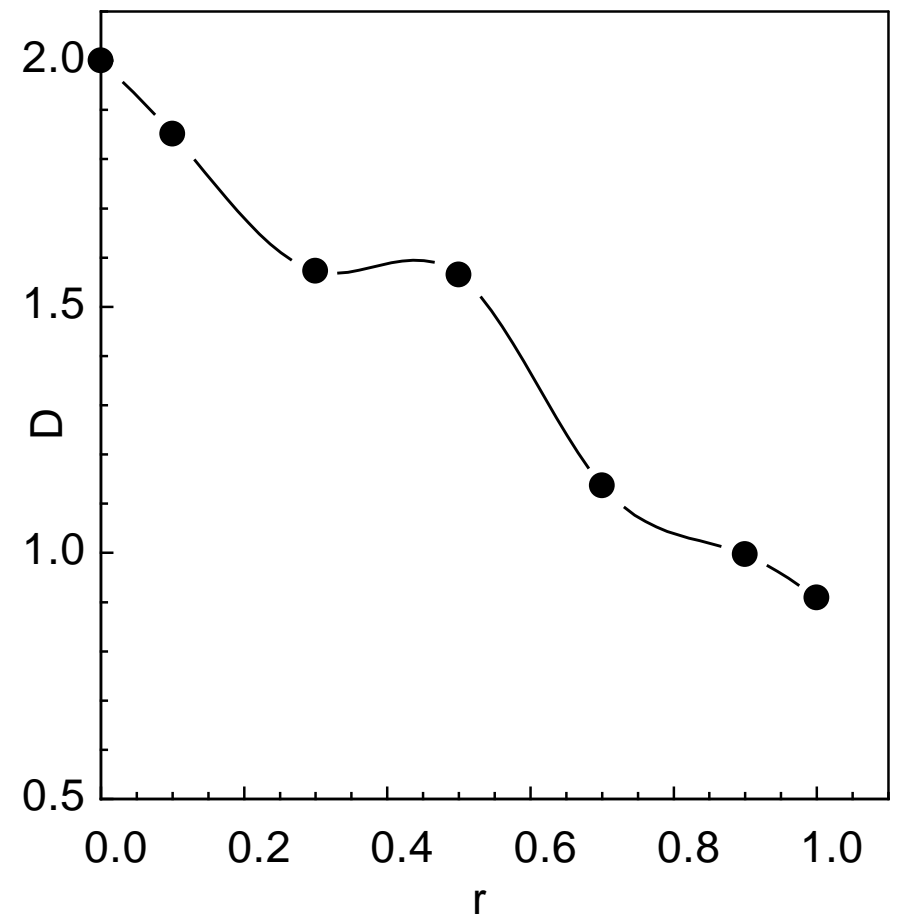


FIGURE-4

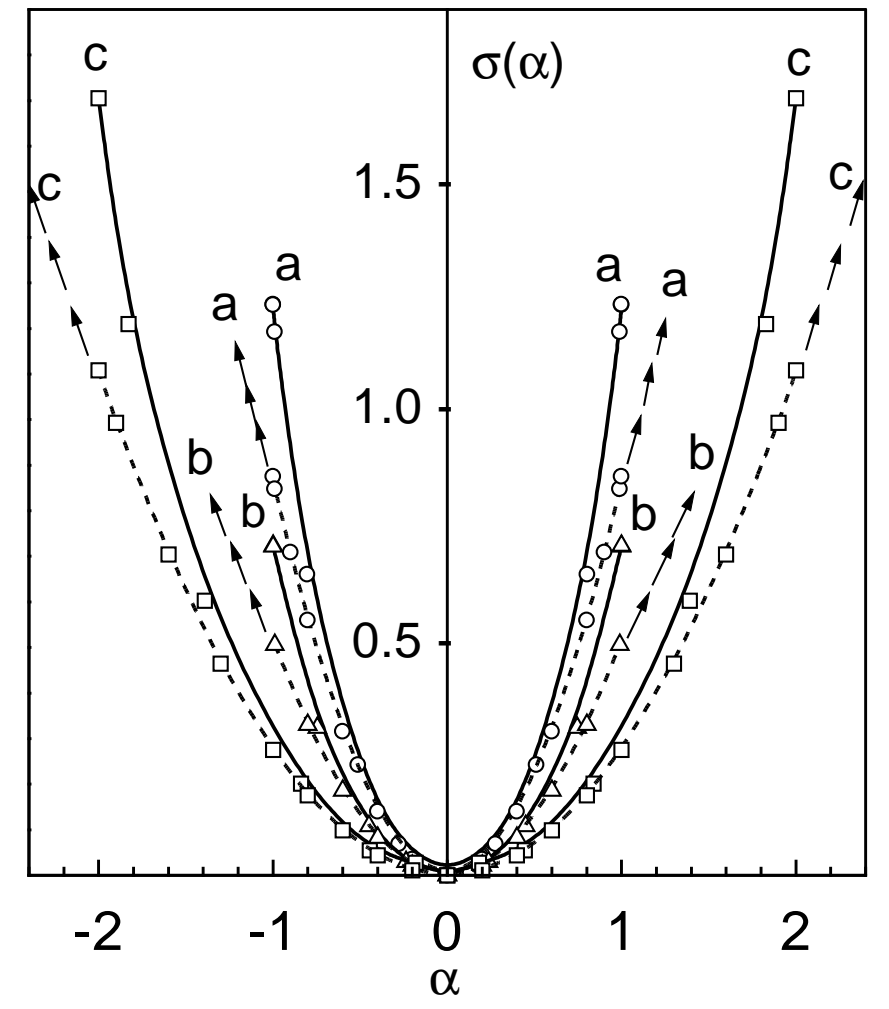

